\title{
Correction to: African evolutionary history inferred from whole genome sequence data of 44 indigenous African populations
}

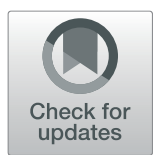

Shaohua Fan 1,2, Derek E. Kelly ${ }^{1}$, Marcia H. Beltrame¹, Matthew E. B. Hansen¹, Swapan Mallick ${ }^{3,4,5}$, Alessia Ranciaro ${ }^{1}$, Jibril Hirbo ${ }^{1,6}$, Simon Thompson? ${ }^{1}$, William Beggs ${ }^{1}$, Thomas Nyambo ${ }^{7}$, Sabah A. Omar $^{8}$, Dawit Wolde Meskel ${ }^{9}$, Gurja Belay ${ }^{9}$, Alain Froment ${ }^{10}$, Nick Patterson ${ }^{4}$, David Reich ${ }^{3,4,5}$ and Sarah A. Tishkoff ${ }^{1,11^{*}}$

\section{Correction to: Genome Biology (2019) 20:82 \\ https://doi.org/10.1186/s13059-019-1679-2}

Following publication of the original article [1], a typographical error in the formula for calculating $d_{i}$ in the "Scans for local adaptation" subsection in the Method section, was identified. The correct formula should be

$$
d_{i}=\sum_{j \neq i}\left(F_{s t}(i, j)-E\left[F_{s t}(i, j)\right]\right) / s d\left(F_{s t}(i, j)\right)
$$

The authors apologize for any confusion this error may have caused.

\begin{abstract}
Author details
'Department of Genetics, University of Pennsylvania, Philadelphia, PA 19104, USA. ${ }^{2}$ Present Address: State Key Laboratory of Genetic Engineering, Human Phenome Institute, School of Life Sciences, Fudan University, 2005 Songhu Road, Shanghai, China. ' ${ }^{3}$ Department of Genetics, Harvard Medical School, Boston, MA 02115, USA. " Broad Institute of Harvard and MIT, Cambridge, MA 02142, USA. ${ }^{5}$ Howard Hughes Medical Institute, Harvard Medical School, Boston, MA 02115, USA. ${ }^{\circ}$ Present Address: Division of Genetic Medicine, Vanderbilt University Medical Center, Vanderbilt University, Nashville, TN 37232, USA. ${ }^{7}$ Department of Biochemistry, Muhimbili University of Health and Allied Sciences, Dares Salaam, Tanzania. ${ }^{8}$ Center for Biotechnology Research and Development, Kenya Medical Research Institute, Nairobi, Kenya. ${ }^{9}$ Department of Biology, Addis Ababa University, Addis Ababa, Ethiopia.

${ }^{10}$ UMR 208, IRD-MNHN, Musée de I'Homme, Paris, France. ${ }^{11}$ Department of Biology, University of Pennsylvania, Philadelphia, PA 19104, USA.
\end{abstract}

Published online: 09 October 2019

\section{Reference}

1. Fan $\mathrm{S}$, et al. African evolutionary history inferred from whole genome sequence data of 44 indigenous African populations. Genome Biol. 2019;20:82. https://doi.org/10.1186/s13059-019-1679-2.

\footnotetext{
* Correspondence: tishkoff@pennmedicine.upenn.edu

'Department of Genetics, University of Pennsylvania, Philadelphia, PA 19104, USA

${ }^{11}$ Department of Biology, University of Pennsylvania, Philadelphia, PA 19104, USA

Full list of author information is available at the end of the article
}

(c) The Author(s). 2019 Open Access This article is distributed under the terms of the Creative Commons Attribution 4.0 International License (http://creativecommons.org/licenses/by/4.0/), which permits unrestricted use, distribution, and reproduction in any medium, provided you give appropriate credit to the original author(s) and the source, provide a link to the Creative Commons license, and indicate if changes were made. The Creative Commons Public Domain Dedication waiver (http://creativecommons.org/publicdomain/zero/1.0/) applies to the data made available in this article, unless otherwise stated. 